\title{
Pilot Study on Following and Resisting Forces on the Pelvis
}

\author{
Jos H. Meuleman, Reinoud Kruithof, Edwin H. F. van Asseldonk, Herman van der Kooij
}

\begin{abstract}
To apply guiding forces in gait training, it is important to know the forces that can be perceived (perception threshold) and the forces a patient cannot resist (resistance threshold). In a pilot study we applied lateral forces on the pelvis by means of a virtual spring on three healthy subjects standing. We measured forces on the pelvis and lateral position on the pelvis. When instructed to follow the exerted forces, the subjects started moving when forces reached $12.5 \mathrm{~N}$. When instructed to resist forces, the subjects were capable of resisting forces up to 40-60 N.
\end{abstract}

\section{INTRODUCTION}

$I^{N}$ $\mathrm{N}$ gait training robots the paradigm is shifting from position control [1] to interaction control [2-4]. The algorithms apply corrective forces based on position or angle errors between target patterns and actual patient performance. To implement interaction control, not only target patterns are required, also knowledge on the effects of corrective forces.

To promote active participation, it is relevant to make a distinction between guiding forces, that give a hint to the patient and enforcing forces, that 'overrule' the patient's contribution [5]. To develop a strategy based on guiding forces, it is important to know how subjects respond to forces.

This paper describes a pilot study on how subjects respond to guiding- and enforcing forces on the pelvis during stance. Results can be used in the development of strategies on balance training during gait [6].

We performed an experiment in which we applied lateral forces to the pelvis of healthy subjects. The subject was instructed either to follow the force or to resist the force. The goal of the tests is to identity a minimum amount of force that makes the subject voluntarily follow the force (perception threshold), and maximum amount of force that the subject can resist (resistance threshold). These thresholds can be used in the design of control strategies for training weight shift and balance control in gait.

\section{METHODS}

We used an admittance controlled servomotor (Moog C40 actuator), connected to the pelvis with a waist strap. The setup is capable of displaying a virtual spring on the pelvis

This study was supported by a grant from Dutch Ministry of Economic affairs and Province of Overijssel, the Netherlands (grant: PID082004).

Jos H. Meuleman is with Moog Robotics, Nieuw-Vennep, The Netherlands (jmeuleman@moog.com).

Jos H. Meuleman, Reinoud Kruithof, Edwin H.F. van Asseldonk and Herman van der Kooij are with the Department of Biomechanical Engineering, University of Twente, Enschede, The Netherlands. (see Fig. 1). The spring stiffness and the position of the base point of the spring were controlled in the experiments.

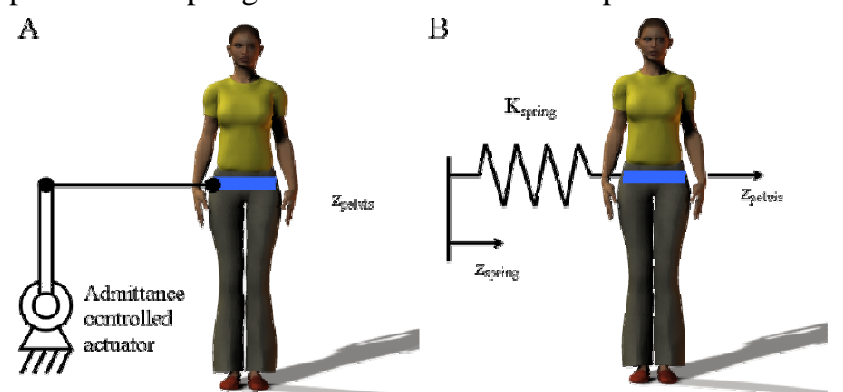

Fig. 1. Setup for applying force to the pelvis. A) Admittance controlled actuator with lever and rod connected to belt. B) Virtual spring attached to the pelvis.

We performed two tests. In the first test, we asked three subjects (length $1.88 \pm 0.03 \mathrm{~m}$; body mass $77.00 \pm 2.65 \mathrm{~kg}$ ) to follow the force that they felt on the pelvis. The guiding force was generated by a virtual spring (see Fig. 1B). The base point of the spring moved randomly to either left or right for $12 \mathrm{~cm}$ in five seconds. The test is considered quasistatic since the motions are slow $(2.4 \mathrm{~cm} / \mathrm{s})$.

When the subject stands still, the force slowly increases until the perception threshold is reached, and then the subject follows the force. In order to assess how well the subjects can follow the force, we used various spring stiffnesses: the subject adapts his pelvis position to minimize the interaction force. Higher spring stiffness, requires either more accurate positioning or results in more fluctuation in the interaction force. In ten trials the following spring stiffness values were used in increasing order: $0,12.5,31.52$, $62.5,125,312.5,625,1250,3125$ and $6250 \mathrm{~N} / \mathrm{m}$.

In the second test the conditions and subjects were equal to the first test, the instruction however was to resist the force. The destabilizing effect of the resistance force is dependent on the base of support and the location of the center of mass relative to the base of support. We instructed the subjects to stand with the feet next to each other and keep the body upright. The force slowly increases, while the subjects stand still. When the resistance threshold is met, the subjects can no longer resist the force and will move with the force.

The subject position and measured force from the tests were recorded at a frequency of $100 \mathrm{~Hz}$. The data was multiplied by the sign of the spring speed, to convert all data to positive values. In the following force test, we defined the perception threshold as the first peak in the measured force, since this indicates the start of following the force.

For the resisting force test a similar approach is taken, 
except that the peak force represents the maximum force the subject can resist. When the peak is reached, the subject cannot resist the force and follows the movement. Consequently the measured force decreases.

\section{RESULTS}

\section{A. Guiding forces - perception threshold}

For lower spring stiffness no clear trend in the response was visible, indicating that the subjects did not feel the force. From stiffnesses of $312.5 \mathrm{~N} / \mathrm{m}$ the perception threshold emerged: the force increased to $10-15 \mathrm{~N}$, then the subject started to follow the spring (Fig. 2 A-D). In most cases the interaction force oscillates around $12.5 \mathrm{~N}$ (Fig. 2 A), however in some cases the force drops to zero or even below zero (Fig. 2 B, subject 1) indicating that the subject moves ahead of the spring. The fluctuation of the force after the threshold is reached appears to be independent of the spring stiffness, the position error decreases with increasing spring stiffness.
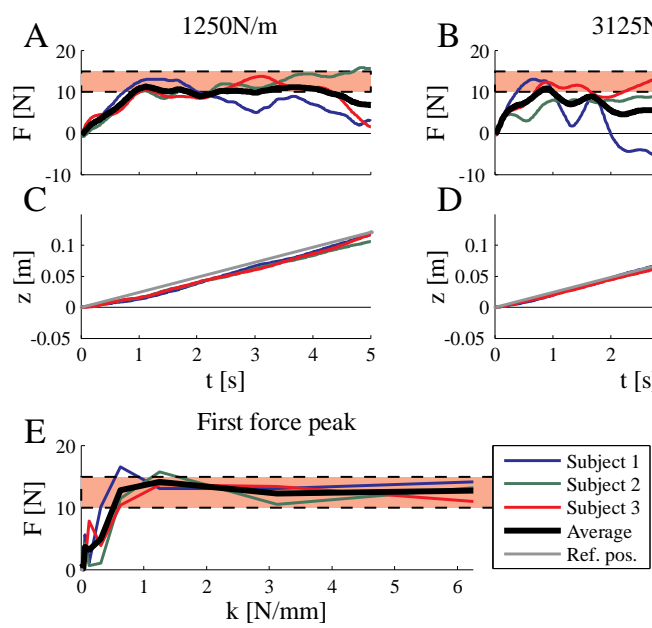

Fig. 2. Responses to an encouraging force on the pelvis, generated by a moving spring: measured force on pelvis with spring stifnesses of $1250 \mathrm{~N} / \mathrm{m}$ (A) and $3125 \mathrm{~N} / \mathrm{m}(\mathrm{B})$; pelvis lateral position response with springstiffness of $1250 \mathrm{~N} / \mathrm{m}(\mathrm{C})$ and $3125 \mathrm{~N} / \mathrm{m}$ (D); first force peaks per trial (E).

\section{B. Enforcing force - resistance treshold}

When resisting soft springs the subjects hardly moved, and thus were able to resist the force. From stiffnesses of $62.5 \mathrm{~N} / \mathrm{m}$ upward resisting became more difficult, resulting in lateral displacement of the pelvis (see Error! Reference source not found. A and B).

Subjects 1 and 3 were able to withstand a force of 40-60 $\mathrm{N}$; when that force was reached, they were forced to follow the spring. Subject 2 showed a larger variance and also lost balance in the $3125 \mathrm{~N} / \mathrm{m}$ trial (Error! Reference source not found. B \& D from $4.2 \mathrm{~s}$ ).

\section{DISCUSSION}

One limitation of our study is that only three subjects participated. The outcomes of the tests are merely an indication. Especially in the resistance tests the body weight and length are of influence on the amount of force a person can resist. However, these results provide sufficient information to build tests where dynamic following- and resisting forces are applied during walking.

A possible explanation for the variance of subject 2 in the resistance test is the trunk orientation. This was not measured, but by leaning towards the force, the subject can resist higher lateral forces. Although the subjects were instructed to stay upright, minor deviations of the trunk orientation may affect the resistance threshold.

Whether the found resisting force threshold also applies for walking is questionable, since the base of support and the dynamics of the center of mass vary.
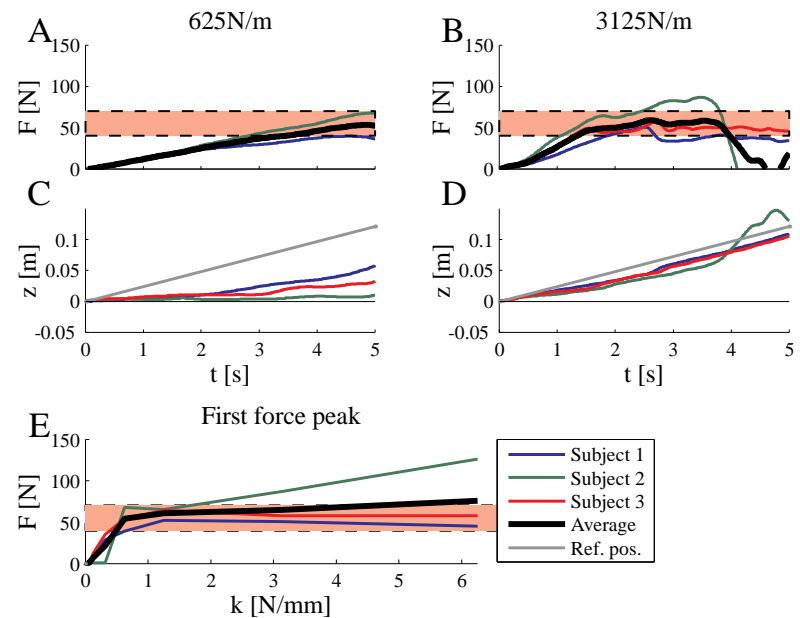

Fig. 3. Responses to resisting force on the pelvis, generated by a moving spring: measured force on pelvis with spring stifnesses of $250 \mathrm{~N} / \mathrm{m}$ (A) and $1250 \mathrm{~N} / \mathrm{m}$ (B); pelvis lateral position response with springstiffness of $250 \mathrm{~N} / \mathrm{m}(\mathrm{C})$ and $1250 \mathrm{~N} / \mathrm{m}(\mathrm{D})$; first force peaks per trial (E).

\section{CONCLUSION}

On the pelvis subjects perceive forces of $12.5 \mathrm{~N}$ upward, and are capable of resisting forces of 40-60N, when both feet are placed next to each other. For gait training this means that when guiding forces on the pelvis are used, they should be $12.5 \mathrm{~N}$ or more. The resistance threshold for walking depends on the timing of the disturbance force, but is expected to have an order of magnitude of $50 \mathrm{~N}$.

\section{REFERENCES}

[1]. Colombo, G., et al., Treadmill training of paraplegic patients using a robotic orthosis. J Rehabil Res Dev, 2000. 37(6): p. 693-700.

[2]. Cai, L.L., et al., Implications of Assist-As-Needed Robotic Step Training after a Complete Spinal Cord Injury on Intrinsic Strategies of Motor Learning. The Journal of Neuroscience, 2006. 26(41): p. 10564-10568.

[3]. Marchal-Crespo, L. and D.J. Reinkensmeyer, Review of control strategies for robotic movement training after neurologic injury. $\mathrm{J}$ Neuroeng Rehabil, 2009. 6: p. 20.

[4]. Reinkensmeyer, D., et al., Tools for understanding and optimizing robotic gait training. J Rehabil Res Dev, 2006. 43(5): p. 657-70.

[5]. Perez, M., et al., Motor skill training induces changes in the excitability of the leg cortical area in healthy humans. Exp Brain Res, 2004. 159(2): p. 197-205.

[6]. Aoyagi, D., et al., A robot and control algorithm that can synchronously assist in naturalistic motion during body-weight- 
supported gait training following neurologic injury. IEEE Trans

Neural Syst Rehabil Eng, 2007. 15(3): p. 387-400. 